\title{
043 IMPLEMENTATION RESEARCH TO SUPPORT THE MINISTRY OF HEALTH AND SOCIAL WELFARE TANZANIA DEVELOP AN EFFECTIVE AND SUSTAINABLE COMMUNITY BASED HEALTH CARE PROGRAM
}

Helen Semu, ${ }^{1}$ Clarence B Mkoba, ${ }^{1}$ Peter Mabwe, ${ }^{2}$ Japhet Killewo ${ }^{2} .{ }^{1}$ Preventive Services, Ministry of Health and Social Welfare, DAR ES SALAAM, Tanzania; 'Epidemiology, Muhimbili University of Health and Allied Sciences, DAR ES SALAAM, Tanzania

\subsection{6/bmjopen-2015-forum2015abstracts.43}

Background To ensure equitable access to health services, Tanzania has maintained a district level integrated facilitycommunity basic health services. The facility component comprised of district hospitals, health centers and dispensaries. Although facility-based service delivery has human resource for Health (HRH) shortages, it is better organized. Since independence in 1961, Tanzania has developed many different types of Community-Based Health Initiatives (CBHI) using voluntary Community Health Workers (CHWs) however, to-date, none of the initiatives has a sustainable plan. The use of paid cadre of CHWs has the potential to be an effective, efficient, sustainable and equitable strategy to deal with the $\mathrm{HRH}$ shortage. An implementation research is paramount from the design to the implementation stages.

Objectives To develop an Implementation research to support program design and implementation.

Methods To work towards a harmonized and integrated Program, the Ministry of Health and Social Welfare (MOHSW) formed a CHW Task Force to advise the Ministry on matters related to development of a National Community Based Health Program (CBHP), Since its creation, a number of initiatives have taken place throughout 2012-2015.

Result A National Policy guideline (2014), Strategic Plan (2014-2020), Training Curriculum (2015) and task sharing guideline were developed, approved and disseminated for use in development of a paid cadre of CHWs that will implement $\mathrm{CBHP}$ and deliver a comprehensive package of intervention that contribute to socio-economic development. A CHWs Learning Agenda Project (CHW-LAP) was initiated to establish a research platform for learning. Through the project, we have created a database that maps geographic and programmatic of CBHI and existing CHWs across the country, A Stakeholder Analysis to elicit stakeholder views on the development and implementation of CBHP and A descriptive costing activity to capture existing programmatic costs.

Conclusion Embedding implementation research in CBHP has provided an opportunity to learn and adjust while implementing the Program activities.

\section{REFERENCES}

1 MOHSW. The National Policy Guideline for Community Based Health Program. Tanzania, 2014.

2 MOHSW. The National Strategic Plan for Community Based Health Program (20142020), Tanzania 2004.

3 MOHSW. The National Curriculum for Basic Technician Certificate in Community Health (NTA level 4) 2015. 